\title{
Creatine Deficiency Syndromes: Diagnostic Pearls and Pitfalls
}

\author{
Claire Hinnell, Michael Samuel, Fadi Alkufri, Keyoumars Ashkan, Yusof Rahman, Charles Turner, \\ R. Neil Dalton, Lina Nashef
}

Can. J. Neurol. Sci. 2011; 38: 765-767

Creatine deficiency syndromes (CDS) are recently described and under-diagnosed inborn errors of creatine metabolism. Clinical features include developmental delay, epilepsy, behavioural problems and movement disorder. Basic laboratory results, such as low serum/plasma creatinine, can suggest these disorders but low-normal 'routine' creatinine levels do not exclude the diagnosis. We describe two sisters who were diagnosed in adulthood with guanidinoacetate methyltransferase (GAMT) deficiency after numerous previous negative investigations. Atypical clinical features in the two siblings expand the phenotype of GAMT deficiency.

\section{Case Reports}

Two female siblings (age 25 and 31 years) were each born at term by spontaneous delivery after unremarkable pregnancies to related Turkish-Cypriot parents. Patient 1 walked at 18 months but began walking on her knees at three years due to a suspected focal dystonia. Speech onset was late and she developed only the use of short sentences and the ability to follow simple commands. She developed epilepsy at 18 months requiring antiepileptic medications. Following intensive physiotherapy, she began walking on her feet again at 15 years. When seen at age 25 years, she had an abnormal gait with flexion at the hips and knees. She had echolalia, had spontaneous output of occasional words and short phrases, and understood simple verbal commands. Behavioural difficulties precluded further detailed examination.

Patient 2, the older sibling of Patient 1 , initially learned to speak in sentences but shortly regressed to single words. Motor milestones were initially normal but at 18 months she began to toe walk. She remained independently mobile and kept up with her peers physically until age 21 years at which time she developed right knee extension. Over the subsequent nine months, the dystonia became generalized to all four limbs, trunk and face. She developed episodes of crying, screaming and agitation. When seen at age 30 years, she had severe generalized dystonia involving all four limbs, trunk, neck, face, jaw and tongue significantly impacting management by her carers. Speech was restricted to occasional single words and verbal comprehension was limited. Prior to aetiologic diagnosis she was considered to have an unidentified secondary or genetic generalized dystonia refractory to medical treatment for which she underwent bilateral internal pallidal deep brain stimulation with standard programming. Within a few days, her involuntary movements had markedly decreased translating into improved sitting tolerance and ease of care. There was no change in cognition or language. Motor improvements have been maintained to the most recent post-operative follow-up at three months, up to which time she had not yet received any other medical treatments for her dystonia or underlying disease.

\section{Results}

Normal or negative previous investigations included: acanthocytes, white cell enzymes (testing for mucolipidosis II and III, GM2 gangliosidosis, mucopolysaccharidosis VII, alpha and beta mannosidosis, fucosidosis, alpha- $\mathrm{N}$-acetylgalactosaminidase deficiency, GM1 gangliosidosis, glucocerebrosidase deficiency, Niemann-Pick disease type A and B, and cholesteryl ester storage disease), copper and caeruloplasmin, amino acids, $\alpha$-fetoprotein, plasma very long chain fatty acids, autoantibodies, Fragile X and Rett's syndrome mutations, urine oligosaccharides and organic acids, magnetic resonance imaging brain, nerve conduction tests, skin and muscle biopsies and mitochondrial mutations. Patient 2 had generalized elevation of urine amino acids.

During routine follow-up, review of laboratory investigations revealed that both sisters had low serum creatinine (by $22 \%$ and $33 \%$ in Patients 1 and 2 respectively when measured with the Jaffe method) suggesting the possibility of CDS. Further analysis of plasma creatinine using stable isotope dilution liquid chromatography electrospray mass-spectrometry (ID LCMSMS) revealed markedly low levels (Table). Additional analysis of plasma and urine creatine and guanidinoacetate using ID LC-MSMS was consistent with GAMT deficiency in both siblings (Table). In retrospect, serum creatinine had been low on several occasions but had not been recognized as a potential clue to diagnosis, being attributed to a poor nutritional state.

From the Department of Neurology (CH, MS, FA, LN), Department of Neurosurgery (KA), King's College Hospital; WellChild Laboratory (CT, RND), King's College London, Evelina Children's Hospital; Guy's and St Thomas' NHS Foundation Trust (YR, CT), London; East Kent Hospitals University NHS Foundation Trust (MS), Ashford, United Kingdom.

Received December 13, 2010. Final Revisions Submitted March 18, 2011 Correspondence to: Claire Hinnell, Foothills Medical Centre, 12th Floor, Neurosciences, 1403 - 29th St. NW, Calgary, Alberta, T2N 2T9, Canada 
Table: Results of laboratory investigations for Creatine Deficiency Syndromes in patients 1 and 2

\begin{tabular}{l|l|l|l}
\hline & Patient 1 & Patient 2 & Normal \\
\hline $\begin{array}{l}\text { Serum creatinine } \\
(\mu \mathrm{mol} / \mathrm{L})\end{array}$ & 35 & 30 & $45-120$ \\
\hline $\begin{array}{l}\text { (Jaffe method) } \\
\text { Plasma creatinine } \\
\begin{array}{l}(\mu \mathrm{mol} / \mathrm{L}) \\
(\mathrm{DD} \mathrm{LC}-\mathrm{MSMS})\end{array}\end{array}$ & 16.2 & 10.8 & $45-120$ \\
\hline $\begin{array}{l}\text { Plasma creatine } \\
(\mu \mathrm{mol} / \mathrm{L})\end{array}$ & 0.3 & 3.4 & $6-50$ \\
\hline $\begin{array}{l}\text { Plasma GAA } \\
(\mu \mathrm{mol} / \mathrm{L})\end{array}$ & 21.6 & 26.2 & $1-3.5$ \\
\hline $\begin{array}{l}\text { Urine GAA } \\
(\mu \mathrm{mol} / \mathrm{mmol} \mathrm{Cr})\end{array}$ & 728 & -- & $3-78$ \\
\hline
\end{tabular}

ID LC-MSMS - isotope dilution liquid chromatography electrospray mass-spectrometry; GAA - guanidinoacetate; $\mathrm{Cr}$ - creatinine

Following diagnosis, both siblings were started on treatment with creatine supplementation $(500 \mathrm{mg} / \mathrm{kg}$ daily in three divided doses). Prolonged follow-up will be required to assess treatment efficacy.

\section{Discussion}

Creatine deficiency syndromes are rare and unfamiliar to most clinicians. Few laboratories offer the appropriate biochemical and genetic testing. Thus these conditions are often unrecognized and under-diagnosed. ${ }^{1}$ An early and accurate diagnose of CDS may lead physicians to offer therapy, and so we emphasize here the clinical features, diagnostic pitfalls and necessary diagnostic investigations.

Three defects in creatine biosynthesis and transport have been described (Figure): (i) deficiency of L-arginine-glycine amidinotransferase (AGAT), (ii) deficiency of GAMT, and (iii) a defect of the creatine transporter (SLC6A8 deficiency), the most commonly reported CDS. ${ }^{2}$

The most common clinical manifestation of CDS is intellectual impairment. ${ }^{3}$ Other core clinical features include developmental delay, epilepsy and behavior abnormalities such as autism. Patients with GAMT deficiency may also suffer from a predominantly extrapyramidal movement disorder. ${ }^{1}$ Our cases have atypical features and contribute to the expanding phenotype of GAMT deficiency. Patient 2 had onset of a severe movement disorder at age 21 years, later than has been previously reported. In a review of 27 cases, movement disorder emerged before age 12 years in all patients, ${ }^{3,4}$ while a subsequent case report described a patient who developed movement disorder at age 17 years. ${ }^{4}$ Patient 1 has better language skills than is typical. Speech delay is almost ubiquitous in GAMT deficiency with patients able to speak only ten words or fewer, irrespective of age and degree of intellectual disability. ${ }^{3,4}$ There is a single case report of a 13 year-old girl with a mild phenotype who was able to speak in short sentences and make good use of grammar. 4

As in our cases, there is often a delay in diagnosis. MercimekMahmutoglu et al reported an onset of clinical manifestations of three months to three years but a mean age of diagnosis of 12.3 years (range 2-29 years) suggesting substantial diagnostic delay in the majority of patients. ${ }^{3}$ Early, accurate diagnosis and treatment have been reported to improve clinical outcomes in some patients. In a review of 27 patients with GAMT deficiency, oral creatine supplementation improved behavior abnormalities in all patients, epilepsy in most patients and movement disorder in half of patients. ${ }^{3}$ Intellectual ability and speech did not respond. ${ }^{3}$ Treatment response appeared more favourable in younger patients. ${ }^{3}$ Additional measures of dietary arginine restriction and ornithine supplementation have been reported to result in further improvement. ${ }^{2}$ Symptomatic therapy remains important and should be offered in conjunction with dietary supplementation. In AGAT deficiency, clinical symptoms also respond to creatine supplementation, but patients with the creatine transporter defect do not seem to respond to treatment, perhaps because the defect does not result in lack of creatine production but rather impaired uptake into the cells. ${ }^{2}$

Serum or plasma creatinine in CDS is usually low and can be a clue to diagnosis. However, a low level may be attributed to poor diet or a non-specific metabolic finding. Importantly, due to lab correction and quantification methods, normal serum creatinine levels may be misleading; serum creatinine measurement methods in routine use tend to over-estimate at

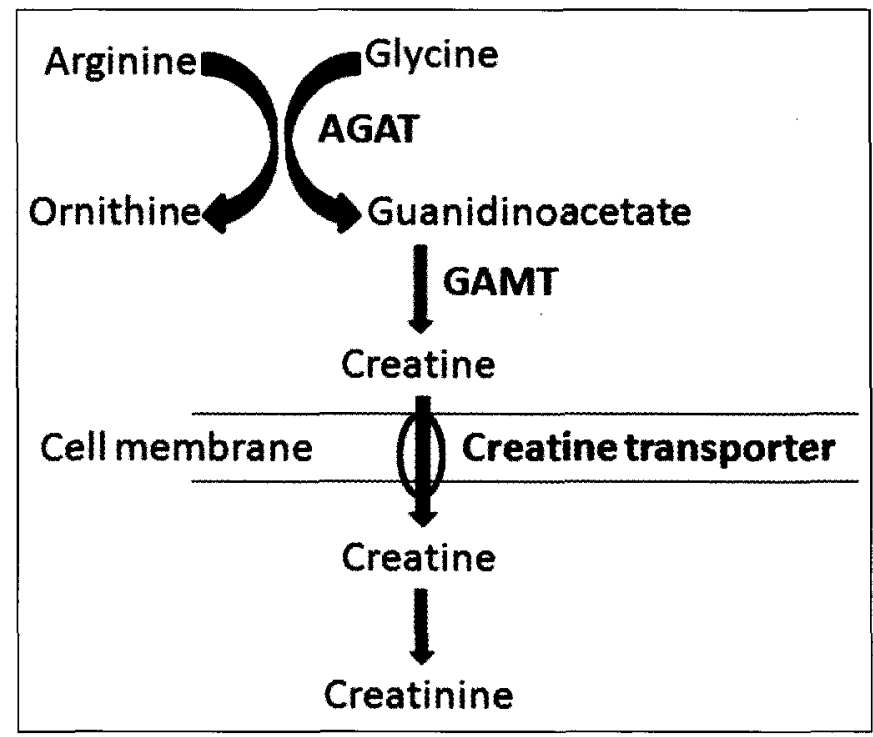

Figure: Schematic of creatine synthesis and transport pathway across the cell membrane. Creatine is synthesized primarily in the liver by two reactions catalysed by the enzymes arginine-glycine amidinotransferase (AGAT) and guanidinoacetate methyltransferase (GAMT). Creatine is then taken up, predominantly by muscle and brain, via the creatine transporter. Creatine is converted to creatinine non-enzymatically and excreted in the urine via the kidneys. 
very low levels. Serum creatinine may appear normal when measured by the Jaffe method but decreased when measured enzymatically, by high performance liquid chromatography or by ID LC-MSMS. Therefore if a laboratory uses the Jaffe method, serum creatinine cannot be used to exclude CDS. ${ }^{5}$ Generalized elevations of urine amino and organic acids, which are falsely elevated when reported with reference to a low urine creatinine, can be another clue to diagnosis but are non-specific. Thus, if CDS is suspected, plasma and urine creatine and guanidinoacetate and urine 24-hour creatinine excretion are recommended. Urine creatinine excretion rate is low in all three types of CDS. Plasma and urine guanidinoacetate allow for differentiation, as levels are high in GAMT deficiency, low in AGAT deficiency and unchanged in SLC68A deficiency. ${ }^{2}$ Proton magnetic resonance spectroscopy may be a useful diagnostic tool, revealing a complete lack of creatine, but the availability of magnetic resonance spectroscopy is limited by cost as well as the need for general anesthetic in patients with intellectual impairment and behavioural problems.

We report two siblings whose clinical features broaden the phenotype of GAMT deficiency. In conclusion, our cases highlight the importance of intermittently re-investigating undiagnosed patients. Creatine deficiency syndromes must be considered in the metabolic differential diagnosis in patients with the common combined phenotype of developmental delay, behaviour problems, epilepsy and/or movement disorder. The clinical variability in presentation makes diagnosis challenging and highlights the need to include CDS on the differential diagnosis of a broad range of symptoms. Consideration should be made for routine screening of children with developmental delay and epilepsy with or without movement disorder by measuring plasma and urine creatine and guanidinoacetate, as serum creatinine alone may be misleading. Earlier recognition of CDS will be advantageous as appropriate therapy may improve certain clinical features, but it remains to be seen if very early treatment can prevent the developmental delay in these conditions.

\section{AUTHOR Disclosures}

M. Samuel has received unrestricted educational grants from Britannia, Solvay, Teva, Ipsen, Boehringer-Ingelheim, Biogen and Medtronic to allow presentations of movement disorders material at conferences. He has received honoraria from Medtronic and UCB. L. Nashef has attended scientific meetings supported by pharmaceutical companies and supervised audits supported by the same.

\section{REFERENCES}

1. Nasrallah F, Feki M, Kaabachi N. Creatine and creatine deficiency syndromes: biochemical and clinical aspects. Pediatr Neurol. 2010 Mar;42(3):163-71.

2. Mercimek-Mahmutoglu S, Stöckler-Ipsiroglu S. Gene Reviews: Creatine Deficiency Syndromes. Gene Reviews 2009 February 2010 [cited July 2010]; Available from: http://www.ncbi.nlm. nih.gov/bookshelf/br.fcgi?book=gene\& part=creatine

3. Mercimek-Mahmutoglu S, Stoeckler-Ipsiroglu S, Adami A, et al. GAMT deficiency: features, treatment, and outcome in an inborn error of creatine synthesis. Neurology. 2006 Aug 8;67(3):480-4.

4. O'Rourke DJ, Ryan S, Salomons G, Jakobs C, Monavari A, King MD. Guanidinoacetate methyltransferase (GAMT) deficiency: late onset of movement disorder and preserved expressive language. Dev Med Child Neurol. 2009 May;51(5):404-7.

5. Verhoeven NM, Guerand WS, Struys EA, Bouman AA, van der Knaap MS, Jakobs C. Plasma creatinine assessment in creatine deficiency: A diagnostic pitfall. J Inherit Metab Dis. 2000 Dec;23(8):835-40. 